\title{
Obturator nerve injury diagnosed by nerve conduction: a case report
}

\author{
Min Cheol Chang ${ }^{1}$, Kyu Tae Choi ${ }^{1}$, Hee Kyung Cho $^{2}$, Yeung Man Kim ${ }^{1}$, Tae Uk Kim ${ }^{3}$ \\ ${ }^{1}$ Department of Rehabilitation Medicine, College of Medicine, Yeungnam University, Daegu, Korea; ${ }^{2}$ Department of Rehabilitation Medicine, \\ Catholic University of Daegu School of Medicine, Daegu, Korea; ${ }^{3}$ Department of Physical Medicine and Rehabilitation, College of Medicine, \\ Dankook University, Cheonan, Korea \\ Correspondence to: Min Cheol Chang, MD. Department of Physical Medicine and Rehabilitation, College of Medicine, Yeungnam University 317-1, \\ Daemyungdong, Namku, Daegu 705-717, Korea. Email: wheel633@ynu.ac.kr.
}

\begin{abstract}
The obturator nerve lies deep within the pelvis, and it can be damaged by direct injury during surgery. In this study, nerve conduction was used to confirm an obturator nerve injury in a patient who presented with hip adductor weakness following gynecological surgery for endometrial cancer. A 56-yearold woman complained of weakness in the right adductor muscles after a laparoscopic hysterectomy due to endometrial cancer. Seven days after surgery, the degree of weakness of the right hip adductor was Medical Research Council (MRC) Scale 1; thus, a nerve conduction velocity test was conducted. To obtain the compound muscle action potentials of the obturator nerve, stimulation was performed $(1.5 \mathrm{~cm}$ inferior and $1.5 \mathrm{~cm}$ lateral to the pubic tubercle) with a surface electrical simulator and recording (midpoint of the right medial thigh) of the adductor muscles. The compound muscle action potentials of the right obturator nerve showed lower amplitude (left side: $2.7 \mathrm{mV}$ vs. right side: $0.3 \mathrm{mV}$ ) and delayed onset latency (left side: $3.2 \mathrm{~ms} v$ s. right side: $2.2 \mathrm{~ms}$ ). These results indicate a partial right obturator neuropathy. Therefore, nerve conduction could be useful to diagnose an early-stage obturator nerve injury and provide information on the degree of damage.
\end{abstract}

Keywords: Obturator nerve injury; nerve conduction; pelvic lymphadenectomy

Submitted Aug 25, 2020. Accepted for publication Nov 19, 2020.

doi: 10.21037/apm-20-1699

View this article at: http://dx.doi.org/10.21037/apm-20-1699

\section{Introduction}

Obturator nerve injury occurs occasionally. Patients with obturator nerve injury usually present with hip adductor weakness and sensory deficits or neuropathic pain in the medial thigh region (1). The known causes of obturator nerve damage include surgery, hemorrhage, tumor compression, and sports-related injuries (1-5). Currently, an obturator nerve injury is diagnosed via physical examination and imaging studies [including magnetic resonance imaging (MRI), computed tomography (CT), or ultrasonography]. However, these techniques are not always effective in detecting nerve injuries (1). MRI and CT are helpful only in situations of mass compression of the obturator nerve, such as that caused by tumors or hematomas. In addition, since the obturator nerve is located deeply, it cannot be examined with ultrasonography in most of its path.

Electromyography (EMG) can be useful to confirm the diagnosis of obturator nerve injury, identifying denervation potentials in the adductor muscles (6). However, EMG abnormalities following obturator nerve injury are only visible after 2-4 weeks (7). Therefore, it is challenging for clinicians to obtain objective evidence for the diagnosis of an obturator nerve injury in the early stages.

Uludag et al. (in 2000) demonstrated the method for nerve conduction of the obturator nerve (8). In that study, the needle with the stimulation electrode was inserted 1-2 cm inferior and 1-2 cm lateral to the pubic tubercle, and the recording electrode was placed on the gracilis muscle belly. However, this method was not used to 
diagnose obturator nerve injuries.

Based on the method from the above study, nerve conduction was used to confirm the diagnosis of obturator nerve injury in a patient who presented with lower limb weakness following surgery for endometrial cancer. We present the following case in accordance with the CARE reporting checklist (available at http://dx.doi.org/10.21037/ apm-20-1699).

\section{Case presentation}

A 56-year-old woman was referred from the obstetric gynecology department to physical medicine and rehabilitation for weakness in the lower right extremity after a laparoscopic hysterectomy for endometrial cancer. The surgery included total laparoscopic hysterectomy, bilateral salpingo-oophorectomy, bilateral pelvic and paraaortic lymphadenectomy, and bilateral para-aortic lymph node dissection. The degree of weakness of the right hip adductor was Medical Research Council (MRC) Scale 1 during the physical examination 7 days after surgery. Weakness of the right hip flexor, extensor, and abductor was not observed. Motor strength in the movement of the right knee and ankle joints was preserved. The knee and ankle jerks were normal; thus, sensory deficits were not assessed. An electrodiagnostic study (Figure $1 A$ ) was conducted on the same day ( 7 days after surgery). To assess nerve conduction of the obturator nerve, the midpoint of the right medial thigh was chosen as the recording site for the adductor muscles. The compound muscle action potentials (CMAPs) of the obturator nerve were recorded with a surface electrode. The stimulation was performed $1.5 \mathrm{~cm}$ inferior and $1.5 \mathrm{~cm}$ lateral to the pubic tubercle site with a surface electrical simulator. The CMAPs of the right obturator nerve showed lower amplitude (left side: $2.7 \mathrm{mV} v$ s. right side: $0.3 \mathrm{mV}$ ) and delayed onset latency (left side: $3.2 \mathrm{~ms} v$ s. right side: $2.2 \mathrm{~ms}$ ) (Figure $1 B$ ) compared to those of the left obturator nerve. The nerve conduction findings for the right femoral, peroneal, and tibial nerves were within normal limits. Needle EMG showed decreased recruitment in the adductor longus, brevis, and magnus muscles; however, denervation potentials were not found. Accordingly, the diagnosis was confirmed to be right obturator neural injury. A pelvic MRI could not detect any nerve abnormality.

In the physical medicine and rehabilitation department, the patient underwent therapeutic exercise to strengthen the adductor muscles and postural balance training to help with standing and walking. At the 6-month follow-up after the onset of weakness, the degree of strength of the right hip adductor improved to MRC 4. The Institutional Review Board of a university hospital approved this study.

All procedures performed in studies involving human participants were in accordance with the ethical standards of the institutional and/or national research committee(s) and with the Helsinki Declaration (as revised in 2013). Written informed consent was obtained from the patient.

\section{Discussion}

In this report, nerve conduction was used to diagnose obturator nerve injury. The CMAPs on the right obturator nerve showed a lower amplitude (11.1\%) and delayed latency $(145.5 \%)$ than those on the left obturator nerve, indicating a partial right obturator neuropathy.

A previous study reported that intraoperative injury to the obturator nerve occasionally occurs during gynecological surgery, mainly due to pelvic lymphadenectomy for uterine or cervical cancer (9). The study reported an incidence rate of obturator nerve injury of approximately $0.7 \%$; however, the difference in incidence rates between open surgical and laparoscopic procedures was not provided (9). In the present case, the obturator nerve was possibly injured during the pelvic lymphadenectomy.

After nerve injury, distal axonal degeneration of the motor fibers occurs within 3-5 days (10). Therefore, CMAP abnormalities begin to appear after approximately 5 days. In the present study, we evaluated the state of the obturator nerve with nerve conduction. This test can provide clinicians and patients with information on the presence and degree of nerve injuries at an early stage. In contrast, denervation potentials appear on EMG 2-4 weeks after nerve injury, and EMG cannot show the extent of neural damage or the nerve conduction velocity (NCV) (7). A previous study reported that sural nerve grafts effectively treated iatrogenic obturator nerve injuries (11). The use of nerve conduction for the diagnosis of obturator nerve injury may help clinicians make an early decision regarding the necessity of nerve grafts for treatment.

In conclusion, nerve conduction could be helpful for the diagnosis of obturator nerve injury. We recommend nerve conduction when patients exhibit symptoms of this specific neural damage, such as weakness of the hip adductor or sensory deficit in the medial thigh. This study is the first to use nerve conduction to diagnose obturator nerve injury; however, this is a single case report, with the associated 

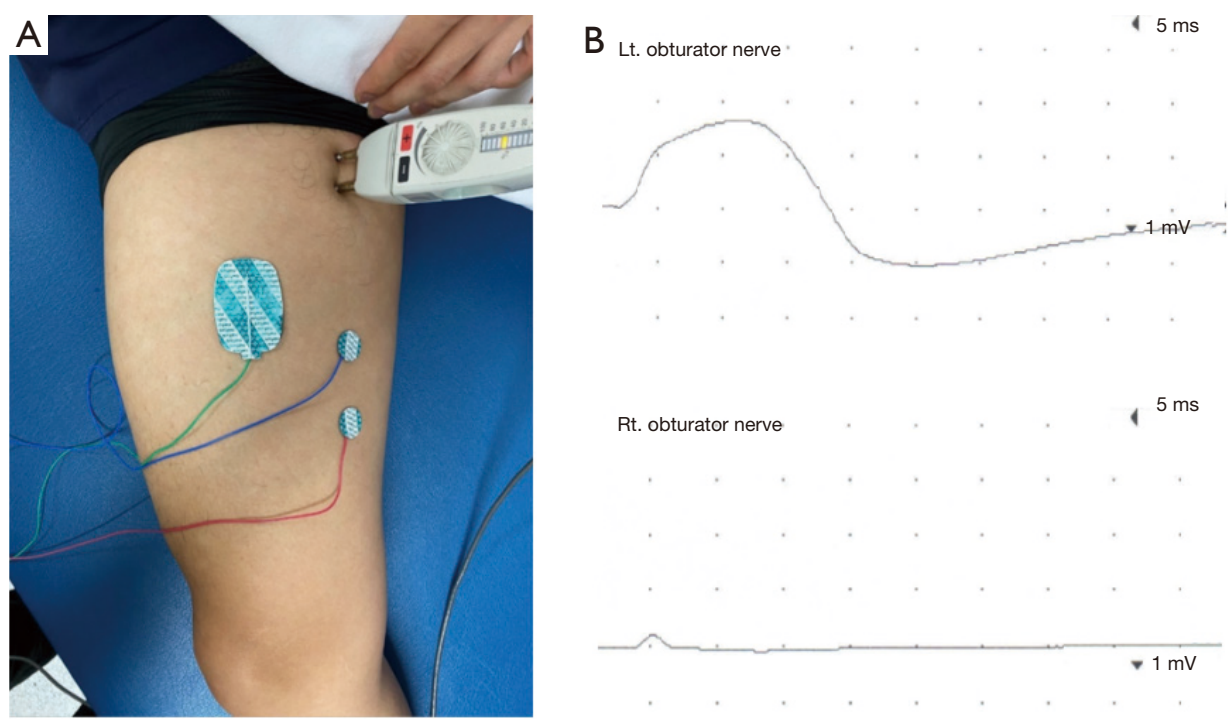

Figure 1 Nerve conduction of the right obturator nerve. (A) The stimulation was performed $1.5 \mathrm{~cm}$ inferior and $1.5 \mathrm{~cm}$ lateral to the pubic tubercle site, and recording was performed at the midpoint of the right medial thigh. (B) Compound muscle action potentials of both obturator nerves showed a difference in amplitude (left side: $2.7 \mathrm{mV}$ s. right side: $0.3 \mathrm{mV}$ ) and onset latency (left side: $3.2 \mathrm{~ms} v$ s. right side: $2.2 \mathrm{~ms}$ ).

limitations. Further studies involving several patients are needed to reach reliable conclusions. In addition, studies to determine the cut-off values (amplitude and latency) for obturator nerve injury are warranted.

\section{Acknowledgments}

Funding: The present study was supported by a National Research Foundation of Korea grant funded by the Korean government (grant number NRF2019M3E5D1A02069399).

\section{Footnote}

Reporting Checklist: The authors have completed the CARE reporting checklist. Available at http://dx.doi.org/10.21037/ apm-20-1699

Conflicts of Interest: All authors have completed the ICMJE uniform disclosure form (available at http://dx.doi. org/10.21037/apm-20-1699). The authors have no conflicts of interest to declare.

Ethical Statement: The authors are accountable for all aspects of the work in ensuring that questions related to the accuracy or integrity of any part of the work are appropriately investigated and resolved. All procedures performed in studies involving human participants were in accordance with the ethical standards of the institutional and/or national research committee(s) and with the Helsinki Declaration (as revised in 2013). Written informed consent was obtained from the patient.

Open Access Statement: This is an Open Access article distributed in accordance with the Creative Commons Attribution-NonCommercial-NoDerivs 4.0 International License (CC BY-NC-ND 4.0), which permits the noncommercial replication and distribution of the article with the strict proviso that no changes or edits are made and the original work is properly cited (including links to both the formal publication through the relevant DOI and the license). See: https://creativecommons.org/licenses/by-nc-nd/4.0/.

\section{References}

1. Tipton JS. Obturator neuropathy. Curr Rev Musculoskelet Med 2008;1:234-7.

2. Barrick EF. Entrapment of the obturator nerve in association with a fracture of the pelvic ring of the pelvic ring. A case report. J Bone Joint Surg Am 1998;80:258-61.

3. Bischoff C, Schönle PW. Obturator nerve injuries during intra-abdominal surgery. Clin Neurol Neurosurg 
1991;93:73-6.

4. Fishman JR, Moran ME, Carey RW. Obturator neuropathy after laparoscopic pelvic lymphadenectomy. Urology 1993;42:198-200.

5. Finan MA, Fiorica JV, Hoffman MS, et al. Massive pelvic hemorrhage during gynecologic cancer surgery: "Pack and go back". Gynecol Oncol 1996;62:390-5.

6. Bradshaw C, McCrory P, Bell S, et al. Obturator neuropathy: a cause of chronic groin pain in athletes. Am J Sports Med 1997;25:402-8.

7. Feinberg J. EMG: myths and facts. HSS J 2006;2:19-21.

8. Uludag B, Ertekin C, Turman AB, et al. Proximal and distal motor nerve conduction in obturator and femoral

Cite this article as: Chang MC, Choi KT, Cho HK, Kim YM, Kim TU. Obturator nerve injury diagnosed by nerve conduction: a case report. Ann Palliat Med 2021;10(6):70697072. doi: 10.21037/apm-20-1699 nerves. Arch Phys Med Rehabil 2000;81:1166-70.

9. Yıkılmaz TN, Öztürk E, Hamidi N, et al. Management of obturator nevre injury during pelvic lymph node dissection. Turk J Urol 2019;45:S26-9.

10. Gagliardo A, Toia F, Maggì F, et al. Clinical neurophysiology and imaging of nerve injuries: preoperative diagnostic work-up and postoperative monitoring. Plast Aesthet Res 2015;2:149-55.

11. Dias AR Jr, Silva E Silva A, Carvalho JP, et al. Correction of iatrogenic injury of the obturator nerve during pelvic laparoscopic lymphadenectomy by the use of sural nerve grafts. Gynecol Oncol Rep 2014;10:16-8 